\title{
Evaluation of plasma damage in blanket and patterned low- $k$ structures by near-field scanning probe microwave microscope: effect of plasma ash chemistry
}

\author{
A.M. Urbanowicz ${ }^{1,2}$, V.V. Talanov ${ }^{3}$, M. Pantouvaki ${ }^{1}$, H. Struyf ${ }^{1}$, S. De Gendt ${ }^{1,2}$, and M.R. Baklanov ${ }^{1}$ \\ ${ }^{1}$ IMEC, Leuven, Belgium \\ ${ }^{2}$ Chemistry Department, KUL, Leuven, Belgium \\ ${ }^{3}$ Solid State Measurements, Inc., Pittsburgh, PA, USA
}

\begin{abstract}
The effect of ash chemistry on dielectric constant of blanket and patterned low- $k$ was studied using a near-field scanning probe microwave microscope, known commercially as NeoMetriK ${ }^{\mathrm{TM}}$ technology. Two common photoresist ash approaches with the same etch sequence were studied: plasma assisted sublimation of photoresist at elevated temperature and ion-assisted ash at room temperature. The results for blanket low- $k$ agree well with the FTIR and water source ellipsometric porosimetry (WEP) measurements. The amount of sidewall damage measured in patterned structures before metallization confirms the expected trends.
\end{abstract}

\section{Introduction}

Plasma induced damage is the main cause of the dielectric reliability loss in low- $k$ materials during the $\mathrm{Cu} / \mathrm{low}-\mathrm{k}$ integration [1]. In order to optimize the patterning process for low- $k$ stacks, the dielectric properties after each processing step have to be evaluated. Typical comb capacitor measurement can only be performed after $\mathrm{Cu}$ metallization [2]. Thus, the obtained dielectric reliability loss is influenced by additional processing steps such as $\mathrm{Cu}$ filling, chemicalmechanical polishing (CMP), and post CMP plasma clean. Since the comb capacitor method is time consuming it may not be used for the real-time process optimization and monitoring. Therefore, it is important to establish an adequate metrology, which provides for rapid evaluation of low- $k$ damage after every processing step without metallization. Then the process can be tuned to further minimize plasma damage while the advanced process control can be implemented in production.

In this paper we present the results obtained using a noncontact dielectric constant metrology based on a near-field scanning probe microwave microscope (NSPMM) [3]. This method allows extracting the effective dielectric constant of low- $k$ material after every processing step on patterned structures without metallization. In order to investigate a feasibility of the microscope for in-line monitoring of pattered low- $k$ stacks we study the effect of ash processes known to result in low and high dielectric damage. Two common approaches have been used: (i) a low temperature, low pressure, anisotropic plasma, where the photoresist is removed by an ion-assisted physical process [4], using oxidizing or reducing plasma chemistries at low temperatures and (ii) an isotropic downstream plasma using a $\mathrm{He} / \mathrm{H}_{2}$ gas mixture where the resist is removed at high temperatures by a thermally activated chemical process $[5,6]$. We additionally evaluated the NSPMM capabilities on blanket wafers with various degrees of ash-induced plasma damage. It is shown that the microwave microscope is a good non-destructive tool for the real-time electrical evaluation of blanket and patterned low- $k$ stacks at every processing step.

\section{Experimental}

\section{A. Sample preparation}

Both blanket (100-nm-thick low- $k$ ) and patterned low- $k$ stacks were deposited on low resistivity $300 \mathrm{~mm}$ Si wafers. Single damascene structures were fabricated by patterning the porogen-based $\mathrm{SiOC}: \mathrm{H}$ materials with $25 \%$ porosity and pristine $k_{\mathrm{p}}=2.5$ as shown Fig.1. Patterning was performed using a $193 \mathrm{~nm}$ resist and targeted at $90 / 90 \mathrm{~nm} \mathrm{~L} / \mathrm{S}$ structures. In this patterning scheme the low- $k$ trenches are etched first and then the photoresist mask is removed by the ash plasmas. All ash conditions were optimized to fully remove the photoresist and are shown in Table 1 . The low- $k$ plus BARC and low- $k$ etch was performed using an industrial capacitively coupled plasma (CCP) reactor for dielectric etch applications. For the etch $\mathrm{CHF}_{3} / \mathrm{CF}_{4}$ 3:16 plasma was applied. The photoresist was stripped using two plasma ash chambers. The first chamber was a high temperature downstream plasma (DSP) reactor for $\mathrm{He} / \mathrm{H}_{2}$ 20:1 anisotropic ash at elevated temperatures. The second one was a CCP reactor with two $\mathrm{N}_{2} / \mathrm{H}_{2}$ and $\mathrm{O}_{2}$ chemistries. We additionally induced different degrees of ash damage to blanket low- $k$ stacks using inductively coupled plasma reactor (ICP) where the plasma of higher density than in CCP reactor produces severe low- $k$ damage [4].

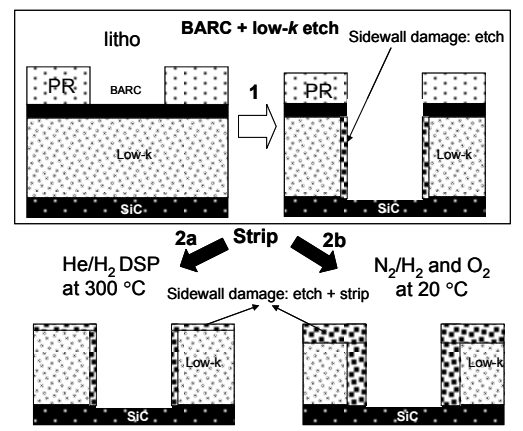

Fig. 1. Photoresist-based etch scheme. Step 1: BARC and low-k etch. Step 2a: plasmas assisted sublimation based strip at elevated temperature. Step $2 \mathrm{~b}$ : ion-assisted strip at room temperature. 
Table 1 Characteristics of used etch and ash processes

\begin{tabular}{|l|l|l|l|l|}
\hline $\begin{array}{l}\text { Wafer } \\
\text { type }\end{array}$ & Treatment & Chamber & Chemistry & Time, s \\
\hline \multirow{3}{*}{ patterned } & Etch & $\mathrm{CCP}$ & $\mathrm{CHF}_{3} / \mathrm{CF}_{4} 3: 16$ & n/a \\
\cline { 2 - 5 } & $\mathrm{Ash}$ & $\mathrm{CCP}$ & $\mathrm{O}_{2}$ & 10,20 \\
\cline { 3 - 5 } & & $\mathrm{CCP}$ & $\mathrm{N}_{2} / \mathrm{H}_{2} 2: 1$ & 30 \\
\cline { 3 - 5 } & & $\mathrm{DSP}$ & $\mathrm{He}_{2} 20: 1$ & 11 \\
\hline blanket & Ash & $\mathrm{ICP}$ & $\mathrm{O}_{2}$ & $10,20,30$ \\
\hline
\end{tabular}

\section{B. Measurement techniques}

Dielectric constant measurements at $4 \mathrm{GHz}$ frequency and ambient conditions were performed by NSPMM on conventional meander-fork structures without metallization. All structures had 50\% pattern density with the width (spacing) ranging from $90 \mathrm{~nm}$ to $150 \mathrm{~nm}$. The schematic for the microwave measurement is shown in Fig. 2a, where the probe is a parallel strip transmission line made of quartz sandwiched between two Al strips. The probe sampling area was about $10 \times 10 \mu \mathrm{m}^{2}$ to address multiple trenches. Because both the "signal" and "ground" probe electrodes are located above the sample and capacitively coupled to it, there is no need to make a contact to or ground the wafer. Fig. 2b shows a lumped element scheme of capacitance measured by the probe, where $C_{\text {ag }}$ describes the probe-sample air gap $\sim 100 \mathrm{~nm}$.

The chemical bonding structure of blanket low- $k$ films before and after plasma treatments was analyzed using a Biorad QS2200 ME FTIR system. The bulk hydrophilicity (amount of adsorbed water) was measured by using WEP (ellipsometric porosimetry with water source) equipped with SENTECH 801 spectroscopic ellipsometer (350-850 nm) [7].

\section{Results and discussion}

\section{A. Blanket wafers}

Several blanket low- $k$ wafers were exposed to $\mathrm{O}_{2}$-based ash plasma in order to induce various degrees of field plasma damage. We used the exposure times of 10, 20, and 30 seconds to induce different amounts of low- $k$ film hydrophilization as shown in Fig. 3 by WEP. In the case of pristine sample the amount of absorbed water is around $1.6 \%$ by volume (at saturated vapor pressure), while the sample treated for $30 \mathrm{~s}$ in $\mathrm{O}_{2}$ plasma absorbs $22 \%$ due to the hydrophilization of pore sidewalls, which nearly equals the total material porosity. Those trends have also been confirmed by FTIR measurement
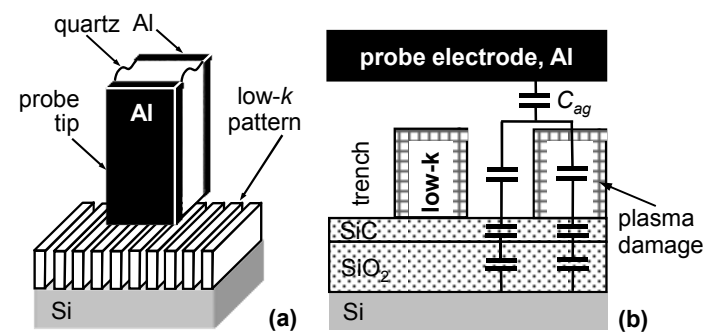

Fig. 2 (a) The probe tip of a near-field scanning probe microwave microscope located above patterned structure. (b) Equivalent scheme of the capacitance measured by the probe.

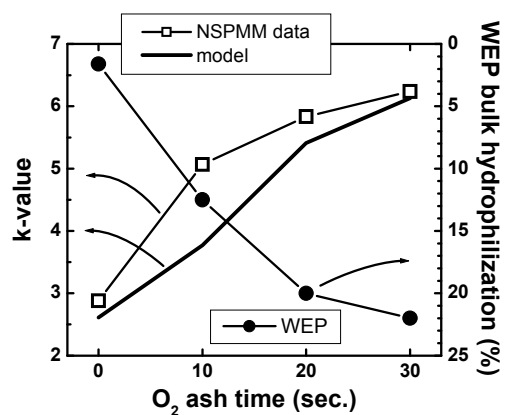

Fig. 3 k-value (as measured by NSPMM) and absorbed water at saturation pressure (as measured by WEP) of blanket low- $k$ films versus time of exposure to $\mathrm{O}_{2}$ plasma in ICP reactor.

(see Fig. 4).

The plasma treatment increases the $k$-value, as measured by the NSPMM (see Fig. 3), because of the following. First, the dielectric constant of low- $k$ matrix increases up to about 4.2 (i.e., that of $\mathrm{SiO}_{2}$ ) due to the carbon depletion. Second, removal of the hydrophobic groups $\mathrm{Si}-\mathrm{CH}_{3}$ from low- $k$ leads to formation of hydrophilic silanol groups, which promotes the moisture adsorption into low- $k$ exposed to air (see Fig. 4). This drastically increases the material average dielectric constant since water has a very large dielectric constant of $k_{\mathrm{H} 20}=75+i 14.5$ at $4 \mathrm{GHz}, 25^{\circ} \mathrm{C}$ [8]. Therefore, considering polarizabilities of all possible bonds formed in low- $k$ after $\mathrm{O}_{2}$ plasma modification, the physisorbed water molecules are mostly responsible for the dipolar contribution to the dielectric loss [9].

For spherically shaped pores the Maxwell Garnett theory [10] yields for the average dielectric constant of porous low- $k$ material:

$k_{a v}=k_{m}\left[1+\frac{3 f\left(k_{\text {por }}-k_{m}\right) /\left(k_{\text {por }}+2 k_{m}\right)}{1-f\left(k_{\text {por }}-k_{m}\right) /\left(k_{\text {por }}+2 k_{m}\right)}\right]$

where $k_{\mathrm{m}}$ is the matrix dielectric constant, $k_{\text {por }}$ is the dielectric constant of the material filling up the pores, and $f$ is the pore fraction. From the WEP data we can estimate the damaged layer thickness as $t_{d}=t_{\text {film }} 100 \mathrm{WEP} / f$, where $t_{\text {film }}$ is the total film thickness, and WEP is the percentage of water by volume (see Fig. 3). Assuming a two layer model for the blanket low- $k$ with a field damage, the dielectric constant of the entire film is:

$k_{\text {meas }}=\frac{k_{a v} k_{p} t_{\text {film }}}{k_{p} t_{d}+k_{a v}\left(t_{f i l m}-t_{d}\right)}$

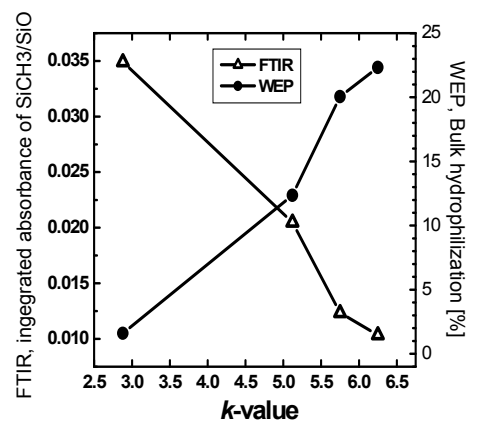

Fig. 4 Correlation between the k-values (as measured by NSPMM) and $\mathrm{SiCH}_{3} / \mathrm{SiO}$ absorbance bands ratio (as measured by FTIR) and bulk hydrophilization (as measured by WEP). 


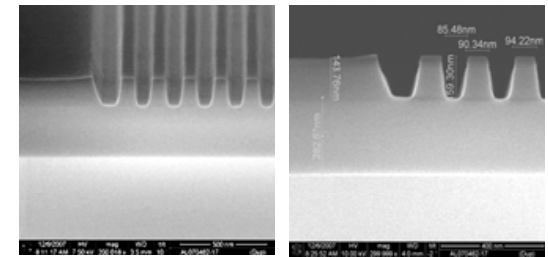

Fig. 5 SEM cross-section of 90/90 $\mathrm{nm} \mathrm{L/S}$ structures after low-k etch and $\mathrm{O}_{2}$ strip: a) tilted view b) no tilt

where $k_{\mathrm{av}}$ is given by (1). The real part of (2) is shown in Fig. 3 by the solid line for $k_{\mathrm{m}}=4.2, k_{\mathrm{por}}=k_{\mathrm{H} 20}, k_{\mathrm{p}}=2.5, f=0.25$, and $t_{\mathrm{d}}$ determined from the WEP measurements. One can see that there is a good agreement between the NSPMM measurements and the model from which we conclude that while the pristine low- $k$ exposed to air retains its electrical properties, nearly all the hydrophilized pores in the plasma damaged low- $k$ become filled up with water.

\section{B. Patterned wafers}

The effective $k$-value of patterned structures (see Fig. 5) as measured by the NSPMM is shown in Fig. 6. The upturn observed at smaller pitches is due to the sidewall damage. Solid lines are the fits to the parallel plate capacitor model of Fig. 2(b) using the damaged layer thickness $t_{\mathrm{d}}$ as the only free parameter [11]. Nominal $k$-values and geometry were assumed for all layers. The dielectric constant of damaged sidewalls was assumed to be 7.6 according to (1) with $k_{\mathrm{m}}=4.2, k_{\mathrm{por}}=k_{\mathrm{H} 20}$, and $f=0.25$. The results agree well with the expected trends. The $\mathrm{O}_{2}$ ash-induced damage increases with the time of the $\mathrm{O}_{2}$ plasma treatment. In the case of $\mathrm{He} / \mathrm{H}_{2}$ DSP strip the sidewall damage is much less as compared with $\mathrm{O}_{2}$ and $\mathrm{N}_{2} / \mathrm{H}_{2}$ CCP. It is well known that $\mathrm{He} / \mathrm{H}_{2}$ DSP strip does not damage low- $k$ while ion assisted strip chemistries result in substantial low- $k$ damage [12]. The observed upturn in $k$-value in the case of $\mathrm{He} / \mathrm{H}_{2}$ DSP strip may be explained by two reasons. The first is the uncertainty of the geometry estimation of the stack, which may also explain a poor agreement between the model and experiment for the $20 \mathrm{~s} \mathrm{O}_{2}$ strip. The second reason may be the damage induced by the trench etch chemistry or interaction of the post trench etch modified layer with $\mathrm{He} / \mathrm{H}_{2}$ ash chemistry.

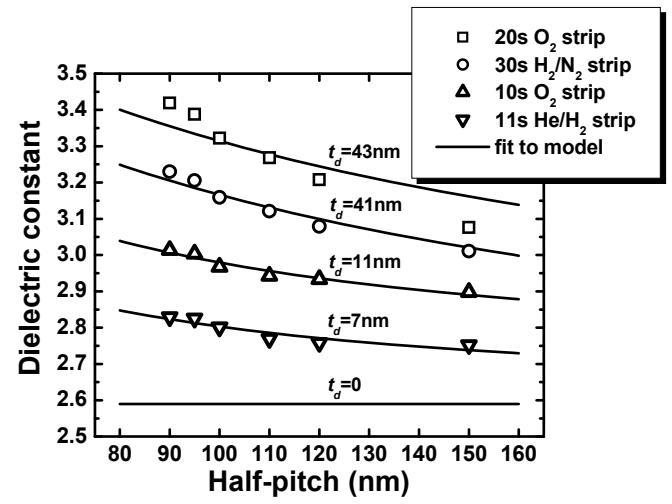

Fig. 6 Effective dielectric constant of patterned structures with $50 \%$ pattern density as measured by NSPMM versus half-pith size for different ash processes. Solid lines are the fits to the parallel plate capacitor model shown in Fig. 2(b) using $t_{\mathrm{d}}$ as the free parameter.

\section{Conclusion}

The dielectric constant of blanket and patterned low- $k$ stacks treated with different plasma ash chemistries has been measured using a near-field scanning probe microwave microscope. We demonstrated that while the pristine low- $k$ exposed to ambient air retains its as-deposited properties, the plasma damaged low$k$ may absorb water into the hydrophilized pores which substantially increases its dielectric constant. The technique is shown to be a good method for non-invasive real time in-line monitoring of patterned low- $k$ structures at every step during interconnect integration.

VVT is grateful to L. Tan and Dr. M.C. Benjamin for assistance with the measurements and useful discussions.

[1] K. Maex, M.R. Baklanov, D. Shamiryan, F. Iacopi, S.H. Brongersma, and Z.S. Yanovitskaya, "Low dielectric constant materials for microelectronics," J Appl. Phys., vol. 93, pp. 87938841, Jun 12003.

[2] Z. Tokei, M. Baklanov, I. Ciofi, L. Younglong, and A. Urbanowicz, "Plasma induced low-k modification and its impact on reliability," Semiconductor Fabtech, pp. 110-115, August 2007.

[3] V.V. Talanov, A. Scherz, R.L. Moreland, and A.R. Schwartz, "Noncontact dielectric constant metrology of low- $k$ interconnect films using a near-field scanned microwave probe," Appl. Phys. Lett., vol. 88, pp. 192906, May 2006;

[4] M. Urbanowicz, A. Humbert, G. Mannaert, Z. Tokei, and M. Baklanov, "Effects of bias, pressure and temperature in plasma damage of ultra low- $k$ films", Sol. State Phenomena, vol. 134, pp. 317-320, 2008.

[5] I.L. Berry, Q. Han, C. Waldfried, O. Escorcia, and A. Becknell, "Use of plasma assisted sublimation to remove photoresist from porous low-k dielectrics," in SEMI® Technical Symposium: Innovations in Semiconductor Manufacturing, 2004.

[6] Q. Han, B. White, I.L. Berry, C. Waldfried, and O. Escorcia, Solid State Phenomena, vols. 103-104, p. 341-344, 2005.

[7] M.R. Baklanov, K.P. Mogilnikov, and Q.T. Le, "Quantification of processing damage in porous low dielectric constant films," Microelectronic Engineering, vol. 83, pp. 2287-2291, Nov-Dec 2006.

[8] J. Barthel, K. Bachhuber, R. Buchner, H. Hetzenauer, and M. Kleebauer, "A computer-controlled system of transmission lines for the determination of the complex permittivity of lossy liquids between 8.5 and $90 \mathrm{GHz}$ ", Ber. Bunsenges. Phys. Chem., vol. 95, pp. 853-859, 1991.

[9] H. Shi, J. Bao, R.S. Smith, H. Huang, J. Liu, P.S. Ho, et al., "Origin of dielectric loss induced by oxygen plasma on organosilicate glass low-k dielectrics," Appl. Phys. Lett., vol. 93, pp. 192909, Nov 102008.

[10] J.C.M. Garnett, "Colours in metal glasses and in metal films," Philos. Trans. R. Soc. London Ser. A, vol. 203, pp. 385-420, 1904.

[11] A.R. Schwartz, V.V. Talanov, A. Scherz, B. Kastenmeier, B. White, and S. Satyanarayana, "Non-destructive electrical characterization of low- $k$ sidewall damage prior to metallization by a near-field scanned microwave probe", 2006 IEEE International Interconnect Technology Conference, June 5-7, 2006, San Francisco, CA, USA.

[12] A.M. Urbanowicz, D. Shamiryan, P. Marsik, Y. Travaly, A. Jonas, P. Verdonck, et al., "Improved low- $k$ dielectric properties using $\mathrm{He} / \mathrm{H}_{2}$ plasma for resist removal", 25th Advanced Metallization Conference, San Diego, CA, 2008. 